\title{
BMJ Open Patterns of change in cardiovascular risks of Korean male workers: a 10-year cohort analysis using the National Health Insurance Service-National Sample Cohort (NHIS-NSC) 2.0 database
}

Hosihn Ryu, Jiyeon Jung (D) , Jihyun Moon

To cite: Ryu H, Jung J, Moon J. Patterns of change in cardiovascular risks of Korean male workers: a 10-year cohort analysis using the National Health Insurance ServiceNational Sample Cohort (NHISNSC) 2.0 database. BMJ Open 2020;10:e038446. doi:10.1136/ bmjopen-2020-038446

- Prepublication history and supplemental material for this paper is available online. To view these files, please visit the journal online (http://dx.doi. org/10.1136/bmjopen-2020038446).

Received 13 March 2020 Revised 08 0ctober 2020 Accepted 12 October 2020
Check for updates

(C) Author(s) (or their employer(s)) 2020. Re-use permitted under CC BY-NC. No commercial re-use. See rights and permissions. Published by BMJ.

College of Nursing, Korea University, Seoul, South Korea

Correspondence to

Dr Jiyeon Jung;

hepburn86@korea.ac.kr

\section{ABSTRACT}

Objective Health behaviour is one of the major determinants of cardiovascular diseases in working population. This study was tried to investigate the trend of cardiovascular health level, the relationship between continuous health behaviours, and changes in the risk of cardiovascular diseases of male workers by using a nationwide database.

Design This study is a retrospective cohort study. Setting and participants The study analysed data of 57837 male workers whose personal health examination data were continuously traced using Korea's National Health Insurance Service-National Sample Cohort 2.0 database.

Primary outcome measures A 10-year trend for all cardiovascular risks and change for the risks according to the consistent performance of healthy behaviours.

Results The results showed that the risk of being overweight (adjusted OR (aOR) 1.63, 95\% Cl 1.59 to 1.68) and obese (aOR 1.51, 95\% $\mathrm{Cl} 1.47$ to 1.56) increased. The index of cardiovascular risk also increased for high fasting glucose (a0R 1.77, 95\% Cl 1.62 to 1.95) and high total cholesterol (aOR 1.68, 95\% $\mathrm{Cl} 1.60$ to 1.76), respectively. The risks of high fasting glucose (aOR $2.09,95 \% \mathrm{Cl} 1.40$ to 3.13 ), high triglycerides (aOR $1.27,95 \% \mathrm{Cl} 1.14$ to 1.42 ) and high low-density lipoprotein cholesterol (aOR 1.38, 95\% Cl 1.14 to 1.66) were increased among high-risk smokers. Similarly, the risk of high total cholesterol (aOR 2.20,95\% $\mathrm{Cl} 1.35$ to 3.58) and high triglycerides (aOR $1.42,95 \% \mathrm{Cl} 1.09$ to 1.85 ) were increased among high-risk drinkers. In addition, the increase in the risk of being overweight (aOR $2.20,95 \% \mathrm{Cl} 1.83$ to 2.65) and obese (aOR 1.90, 95\% $\mathrm{Cl} 1.59$ to 2.27) were analysed among who had not consistently exercised. Conclusions Since the pattern of change in the level of cardiovascular risk related to the continuous health behaviours of male workers was identified, the findings of the present study can be used as basic data to develop health promotion policies for the population.

\section{INTRODUCTION}

The rapid increase in the prevalence of chronic diseases is becoming a major reason
Strengths and limitations of this study

- The study analysed nationwide health examination database of a same working population in Korea continuously tracked once a year.

- This study differs in that it analysed the effects of continued health behaviours on the cardiovascular disease risks of male workers.

- The data analysed in this study may underestimate the risk of cardiovascular disease due to the lack of detailed clinical information in the health examination database.

- The external validity of the results may be open to contention because all subjects were members of the Korean working population.

for the increasing disease burden globally. ${ }^{1}$ Cardiovascular diseases (CVDs) are a major cause of about $50 \%$ of deaths and about $25 \%$ of work disability among the working population worldwide. ${ }^{2}$ In addition, $10-20 \%$ of all causes of CVD deaths among the working population are work related, and the loss of labor due to CVDs is gradually increasing. ${ }^{3}$

Management for relevant risk factors is important for preventing CVDs among the working population. However, the treatment rate and complication management of the diseases that precede CVDs, such as hypertension, dyslipidaemia, diabetes and obesity, are still low. ${ }^{4}$ Furthermore, it has been shown that the proportion of smoking and highrisk drinking among male workers is higher than among general adult males, and male workers have health-damaging features such as job stress. ${ }^{5}$ The formation and retention of healthy behaviours during working age, the longest life phase, are also crucial for preventive health management. However, 
recent policies for the improvement of workers' health and health behaviours have not been effectively implemented, and even basic data on workers' health status to advocate for policy change are insufficient. ${ }^{6}$

Given the current situation, our research team conducted a cross-sectional study analysing the relationship between socioeconomic status and the CVD risk of workers. ${ }^{7}$ On the basis of the findings, the present study was conducted, a 10-year cohort analysis on the trends in health level related to CVDs among male workers using the National Sample Cohort 2.0 database (NSC 2.0 DB) from Korea's National Health Insurance Service (NHIS). A retrospective cohort analysis was performed to track the general annual health examination data of the same workers for 10 years to investigate the relationship between the health behaviours of male workers and their health level in relation to CVDs. The cohort study, a widely used research method, provides an important source of information to identify the major determinants of CVDs by showing the influence of health behaviours on health results. ${ }^{8}$

A Swedish cohort study ${ }^{9}$ confirmed that the risk of heart failure was reduced among those who practised healthy behaviours consistently. In addition, a Japanese cohort study suggested the importance of healthy behaviours for CVD prevention. ${ }^{10}$ In Korea, a regional study determined the relationship between health behaviours and the risk of all-cause premature death ${ }^{11}$ and analysed the relationship between health behaviours and CVD death rates. ${ }^{12}$ However, no study has been conducted as an annual retrospective cohort on the same large group to comprehensively understand the relationship between continuous health behaviours and health level related to CVDs among male workers.

Thus, the present study aimed to provide basic data for workers' health promotion policies through its examination of changing cardiovascular risk patterns among male workers. This was achieved by investigating the trend of health levels, the relationship between continuous health behaviours and changes in the risk of CVDs of male workers using a 10-year cohort analysis at the national level.

\section{METHODS}

\section{Data source}

The present study was a retrospective cohort study conducted using the NHIS-NSC 2.0 DB. In South Korea, universal healthcare coverage was established in $1989 .{ }^{13}$ In $2016,50.76$ million people ( $97.1 \%$ of total population) were covered by National Health Insurance, of which 36.68 million people $(72.3 \%)$ had employee health insurance. ${ }^{14}$ The NHIS implements various health examinations on National Health Insurance subscribers at each life stage and has accumulated the data to build the National Health Information Database (NHID). Since the NHID includes the entire population of South Korea, the data provide statistically meaningful results, and the nation's health level can be analysed accurately.
The NSC 2.0 DB was built by sampling about $2 \%$ (1 000 000 people) from the NHID population. The eligible population was stratified with proportional allocation according to age, sex, region, health insurance type (subscriber classification) and household income (premium bracket). Within each stratum, systematic stratified random sampling was conducted using the individual's total annual medical expenses. To ensure representativeness of the cohort, the sample was evaluated by examining that the CI for the average total annual medical expenses contained the population average. Furthermore, the sample were compared with the population by residence distribution and average of premium. The NSC 2.0 DB comprises data on 'qualification and premium,' 'birth and death,' 'medical treatment,' 'health examination' and 'medical care institution'. ${ }^{16}$ The present study used the Qualification and Health Examination databases of the NSC 2.0 DB to investigate the relationship between health behaviours and the risk of CVDs. An individual's health examination information is included only if the individual, among the samples extracted from the Qualification database, had had a health examination; such individuals comprised around 17\% of the entire NSC 2.0 DB in 2006.

\section{Study participants: the cohort of the study}

This study participants were limited to those with employee insurance according to the subclassification code of the insurance type in the qualification database. The data of 1108369 insured people, excluding dependents of the insurance were analysed. After tracking people who had employee insurance continuously for 10 years (from 2006 to 2015), 1006888 people who were not included in the employee code even for 1 year during the period were excluded from the analysis. Second, 720 people classified as employees but who were outside working age (15-64 years) were excluded. Of the remaining 100761 people, 19191 people who had medical history and medication for hypertension, diabetes, stroke, heart disease or hyperlipidaemia were excluded. The cohort of the study was thus composed of 57837 healthy male workers with no history of hypertension, diabetes, hyperlipidaemia and CVD and no history of use of cardiovascular or antidiabetic drugs (see online supplemental file 1 ).

\section{Patient and public involvement}

In this study, we analysed retrospective data from the NHIS. We followed the operating regulations of NHIS to analysis the database. No additional patients or participants were recruited for this study. Thus, the study did not require the consent of individuals in accordance with the research guidelines and ethics of Institutional Review Board and NHIS.

\section{Measurements}

For the analysis of the targets' general characteristics, gender, age, region and household income were collected from the qualification database. The participants' residence districts were categorised into four groups based on 
equality of population distribution: Seoul (capital city), metropolitan cities other than Seoul, Gyeonggi-do, which is an area (capital province) near Seoul, and other provinces. Household income was calculated using premium brackets comprising 10 quantiles and was classified into five groups: 1-2 quantile, $3-4$ quantile, $5-6$ quantile, $7-8$ quantile and $9-10$ quantile, with a higher premium quantile indicating a higher income level.

Some data from the health examination and health behaviour variables were analysed for CVD risk factors. The American Health Association defines ideal cardiovascular health by the presence of favourable health features (nonsmoking, ideal body mass index (BMI), physically active and good diet) and three favourable health factors (within ideal range of fasting glucose (FG), total cholesterol (TC) and blood pressure (BP)). ${ }^{17}$ Thus, we included BMI, waist circumference (WC), BP, FG, TC, triglycerides (TGs), highdensity lipoprotein cholesterol (HDL-C) and low-density lipoprotein cholesterol (LDL-C) in this study. These indices reflect four important physiometabolic changes related to CVD risks, namely, elevated $\mathrm{BP}$, increased weight, hyperglycaemic and dyslipidaemia. ${ }^{12}$ Of these, WC has been available in the data source since 2008, and TG, HDL-C and LDL-C have been available since 2009.

BMI was used to define obesity. According to the Asian-specific criteria of obesity, body weight was classified into normal (BMI 18.50-22.99), overweight (BMI 23.00-24.99) and obese (BMI $\left.\geq 25.00 \mathrm{~kg} / \mathrm{m}^{2}\right) .{ }^{18}$ If WC was $90 \mathrm{~cm}$ or greater, it was classified as abdominal obesity. ${ }^{19}$ BP was classified as normal $(<120 / 80 \mathrm{~mm} \mathrm{Hg})$, borderline $(\geq 120 / 80 \mathrm{~mm} \mathrm{Hg})$ or hypertensive $(\geq 140 / 90 \mathrm{~mm}$ $\mathrm{Hg}){ }^{20} \mathrm{FG}$ was classified as normal $(<100 \mathrm{mg} / \mathrm{dL})$, pre-diabetes (100-125 mg/dL) or high FG ( $\geq 126 \mathrm{mg}$ / dL). ${ }^{21}$ Dyslipidaemia is defined as a condition that satisfies one or more of such conditions: high-risk TC if $\mathrm{TC} \geq 240 \mathrm{mg} / \mathrm{dL}$, high-risk $\mathrm{TG}$ if $\mathrm{TG} \geq 200 \mathrm{mg} / \mathrm{dL}$, low HDL-C if HDL-C $<40 \mathrm{mg} / \mathrm{dL}$ and high LDL-C if LDL-C $\geq 160 \mathrm{mg} / \mathrm{dL}$. $^{22} 23$

Health behaviour variables, including smoking, drinking and physical activities, were collected from the Health Questionnaire database. Smokers were classified into non-smokers, past smokers and current smokers. The data collected comprised amount of smoking per day and duration of past smoking and current smoking year. For drinking, drinking frequency and quantity were surveyed. Up until 2008, smoking and drinking were measured using multiple-choice items, with essay format measurements beginning in 2009. Physical activity was measured by the average number of days for overall activities per week until 2008; from 2009, the average number of days per week was measured for 'walking,' 'moderate physical activity,' and 'high-intensity physical activity.' We used the following definitions for measuring physical activity: moderate activity is any activity that makes one's heart rate a little faster than usual, for more than $30 \mathrm{~min}$ a day, and high-intensity physical activity is any intense activity that makes one's breathing and heart rate much faster than usual, for more than $20 \mathrm{~min}$ a day. ${ }^{24}$
In addition, the following definitions were constructed to compare the progress of health risks between those who consistently practised healthy behaviours and those who practised unhealthy behaviours. The participant was defined as a non-smoker or a high-risk smoker depending on whether they had been a non-smoker or a smoker for 10 consecutive years, respectively. The participant was defined as a non-drinker or a high-risk drinker depending on whether they had been sober or had drunk seven drinks or more per week for 10 consecutive years, respectively. ${ }^{24}$ Regular physical activity was defined as physical activity of $15 \mathrm{~min}$ or longer per session and at least three times per week, following the American College of Sports Medicine guidelines. The participants were classified into 'non-exerciser' and 'healthy exerciser' according to the definition, ${ }^{25}$ and their data were then analysed.

\section{Statistical analysis}

The data were analysed using SAS V.9.3. Descriptive statistics were performed to determine the general characteristics, CVD health level and the distribution and trends in the level of health-related behaviours of male workers in the past 10 years. The trend in health level was presented in terms of prevalence rate as a result to show changes in health through the years of follow-up for the same subject. The linear regression methods for trend estimation was performed to describe the change in health status of the participants.

In particular, homogeneity tests were performed on the adjustment variables of age, residence location and income level using the $\chi^{2}$ test. Multilevel logistic regression analyses, which can consider two levels simultaneously, were performed to analyse the 10-year change of each risk, using the GENMODE procedure of SAS V.9.3. Multilevel logistic regression analyses were performed using age, residence location and income level as the adjustment variables to calculate the change of CVD risks per year and CVD risks according to the performance of healthy behaviours. The mixed procedure of SAS V.9.3 was used to achieve this. The OR for the performance of healthy behaviours concerning CVD risks was evaluated after adjusting for potential confounders (age, region and income).

\section{RESULTS}

\section{Sociodemographic characteristics of the participants}

The baseline year (2006) characteristics of the study cases $(\mathrm{n}=57837)$ were as follows. The most frequent age was $30-39$ years $(26734,46.22 \%)$ followed by $40-49$ years (17 659, 30.53\%), 20-29 years $(9088,15.71 \%), 50-59$ years $(3888,6.72 \%), 60-64$ years $(453,0.78 \%)$ and $15-19$ years $(15,0.03 \%)$. Residence locations (region) were Seoul (capital city; 12228 participants, $21.14 \%$ ), metropolitan cities other than Seoul (14734 participants, 25.48\%), Gyonggi-do, an area that is near Seoul (capital province; 13793 participants, 23.85\%) and other areas (other province; 17082 participants, 29.53\%). There were $1764(3.05 \%)$ participants in the income bracket of 

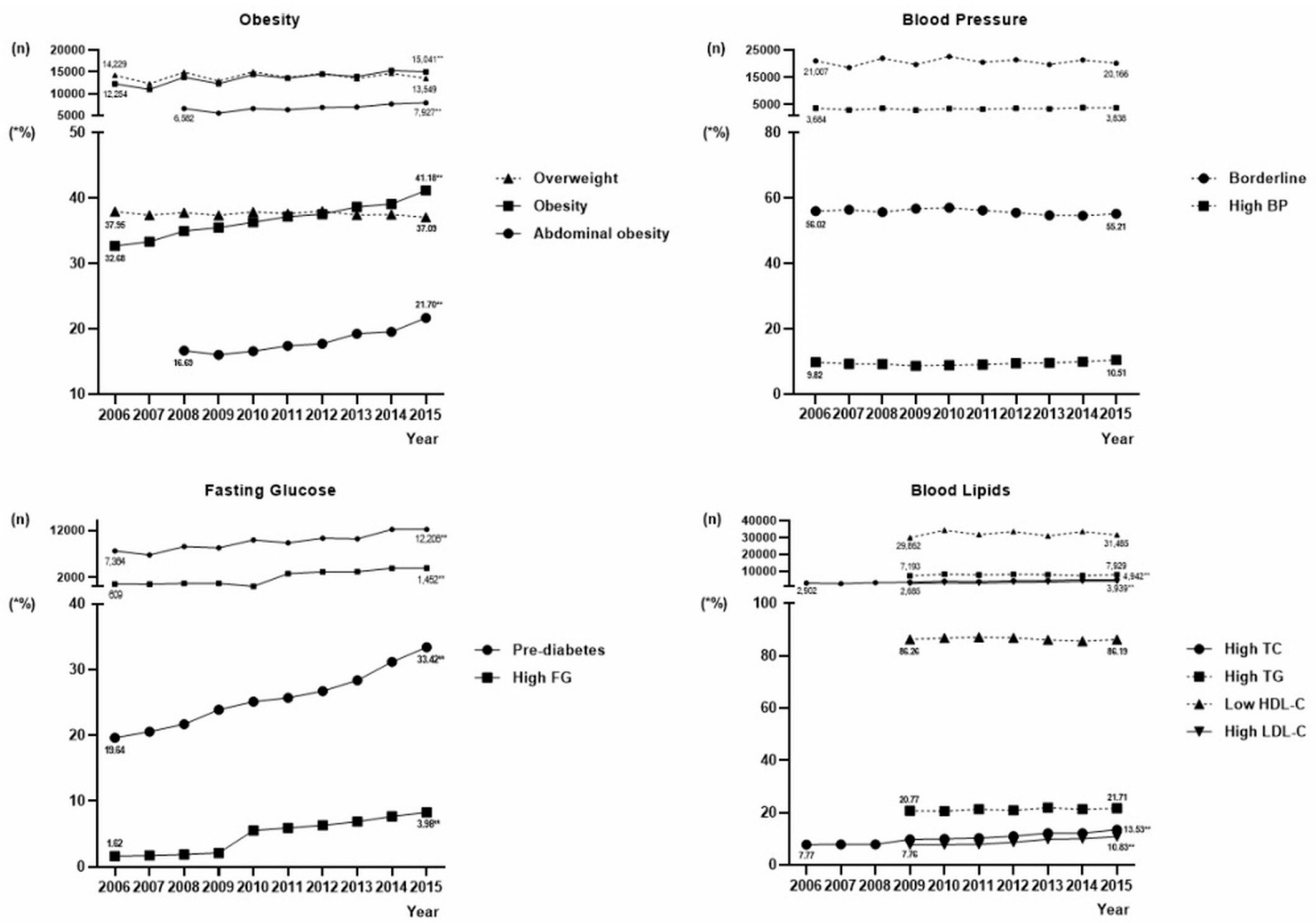

Figure 1 Trends in health status among the participants ( $n=57837)$. Overweight (BMI 23.00-24.99); Obesity (BMI $\geq 25.00 \mathrm{~kg} /$ $\left.\mathrm{m}^{2}\right)$; Abdominal obesity $(\mathrm{WC} \geq 90 \mathrm{~cm})$; Borderline BP $(\geq 120 / 80 \mathrm{mmHg})$; High BP $(\geq 140 / 90 \mathrm{mmHg})$; Prediabetes $(100-125 \mathrm{mg} / \mathrm{dL})$; High FG ( $\geq 126 \mathrm{mg} / \mathrm{dL}) ;$ High TC ( $\geq 240 \mathrm{mg} / \mathrm{dL}) ;$ High TG ( $\geq 200 \mathrm{mg} / \mathrm{dL})$, Low HDL-C ( $<40 \mathrm{mg} / \mathrm{dL})$, and High LDL-C ( $\geq 160 \mathrm{mg} /$ $\mathrm{dL}$ ). ${ }^{*} \%$ prevalence rate among total number of cases in each cell, ${ }^{* *} \mathrm{p}$-value for trend $<0.01$. BMI, body mass index; BP, blood pressure; Fg, fasting glucose; HDL-C, high-density lipoprotein cholesterol; LDL-C, low-density lipoprotein cholesterol; TC, total cholesterol; TG, triglycerides; WC, waist circumference

the 1-2 quantile, $4754(8.22 \%)$ in the $3-4$ quantile, 7613 $(13.16 \%)$ in the 5-6 quantile, $10532(18.21 \%)$ in the $7-8$ quantile and $17283(29.88 \%)$ in the $9-10$ quantile.

\section{Trends in health status of the participants}

The analyses of participants' health-level trends for 10 years are presented in figure 1 . The trends for obesity showed that the prevalence for being overweight was maintained at around $37 \%$, while the prevalence of obesity increased from $32.68 \%$ to $41.18 \%$. The trends of abdominal obesity also showed an increase, from $16.69 \%$ in 2008 to $21.70 \%$ in 2015 . With regard to FG, the proportion of participants corresponding to the pre-diabetic stage increased from $19.64 \%$ to $33.42 \%$, while the prevalence of the higher level of FG corresponding to diabetes increased from $1.62 \%$ to $3.98 \%$. With regard to dyslipidaemia, the ratio of high-risk TC $(\geq 240 \mathrm{mg} / \mathrm{dL})$ and high LDL-C $(\geq 160 \mathrm{mg} / \mathrm{dL})$ increased from $7.77 \%$ to $13.53 \%$ and from $7.76 \%$ to $10.83 \%$, respectively.

\section{Ten-year CVD risks of the participants}

Figure 2 displays the analysis of changes in the CVD risks among the participants $(n=53837)$ by year. The results of the analysis after adjusting for age, region and income level showed that participants' risk of being overweight increased by 1.63 times in 2015 compared with that in 2006 (95\% CI 1.59 to 1.68$)$ and that the risk of obesity also increased by 1.51 times (95\% CI 1.47 to 1.56 ). Risk of abdominal obesity increased by 1.53 times in 6 years from 2009 to 2015 (95\% CI 1.46 to 1.58). Risk of diabetes had the highest increase, of about 1.77 times $(95 \%$ CI 1.62 to 1.95 ), with 1.68 times (95\% CI 1.60 to 1.76 ) increase in the high risk of TC among the dyslipidaemia indices. The analysis showed an overall increase in CVD risk indices.

The risks of hypertension decreased somewhat, by 0.90 times (95\% CI 0.86 to 0.94$)$, respectively.

\section{Ten-year CVD risks by continuous health behaviours}

Changes in the risks of high-risk smokers and non-smokers for 10 consecutive years

Figure 3 presents the analysis of the CVD risks among male workers $(\mathrm{n}=6585)$ who have been either smokers or non-smokers for 10 consecutive years. The risks of obesity, diabetes, high TC, high TG and high LDL-C were statistically significant among persistently smoking male workers.

Adjustments for age, region and income level showed that the risk of high level of FG increased by 2.09 times (95\% CI 1.40 to 3.13 ) among persistent smokers. 

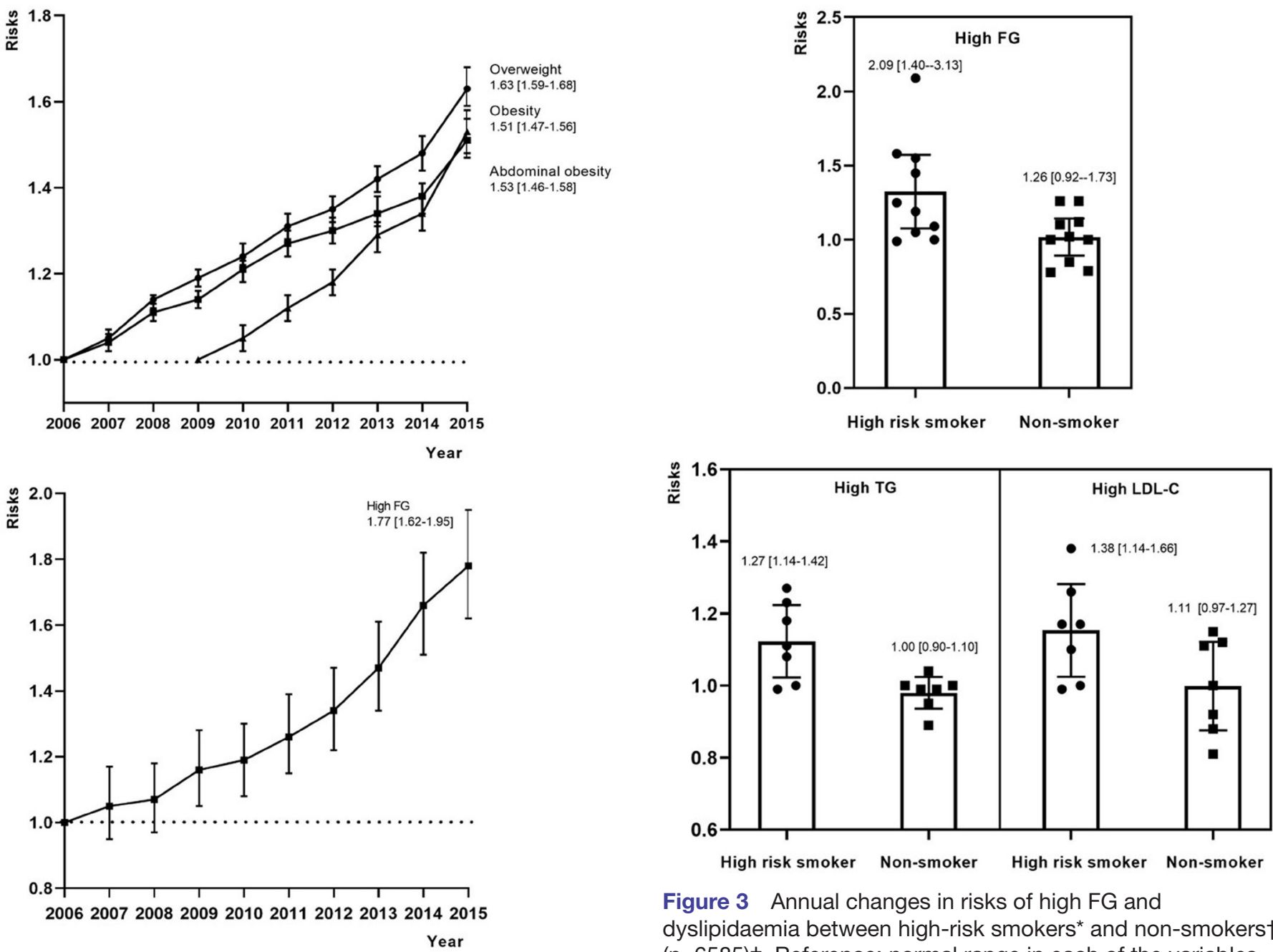

Figure 3 Annual changes in risks of high FG and dyslipidaemia between high-risk smokers ${ }^{*}$ and non-smokers $\dagger$ $(n=6585) \neq$. Reference: normal range in each of the variables.

Figure 2 Annual changes in risks of obesity and high FG among the participants $(n=53837)^{\star}$. Reference: normal range in each of the variables. *Adjusted for age, region, income. FG, fasting glucose.

Increases in major indices, such as the risk of a high level of TG (1.27 times; 95\% CI 1.14 to 1.42$)$ and LDL-C (1.38 times; $95 \%$ CI 1.14 to 1.66 ) were also found; however, the risk of obesity (BMI and WC) and TC increased for both smokers and non-smokers even though the levels of risk were somewhat different.

Changes in the risks of high-risk drinkers and non-drinkers for 10 consecutive years

Figure 4 presents the risks for CVDs among male workers ( $n=1330)$ who had been either high-risk drinkers or nondrinkers for 10 consecutive years. After adjusting for age, the workers who had been persistent high-risk drinkers had a risk of high TC that increased by 2.20 times (95\% CI 1.35 to 3.58), and the risk of high TG increased by 1.42 times (95\% CI 1.09 to 1.85$)$. In addition, obesity increased by 1.59 times ( $95 \%$ CI 1.25 to 2.03 ) for high-risk drinkers. However, the increase in the risk of abdominal obesity was somewhat higher among non-drinkers than among highrisk drinkers.

Changes in the risks of healthy exercisers and non-exercisers for 10 consecutive years

With regard to male workers who did not perform healthy walking consistently for 10 consecutive years, the risk of being overweight and of obesity increased by 2.20 times (95\% CI 1.83 to 2.65) and 1.90 times (1.59-2.27), respectively. Among workers who did not perform moderate exercise for 10 consecutive years, the risks of being overweight and of obesity increased by 2.04 times (95\% CI 1.83 to 2.27 ) and 1.75 times (95\% CI 1.59 to 1.92), respectively. In addition, among those who did not engage in high-intensity exercise for the past 10 consecutive years, the risks of being overweight and of obesity increased by 2.02 times (95\% CI 1.83 to 2.23 ) and 1.66 times (95\% CI 1.52 to 1.83 ), respectively (see figure 5 ).

\section{DISCUSSION}

The present study performed a 10-year cohort analysis of the trends of 10-year CVD risk factors, as well as 

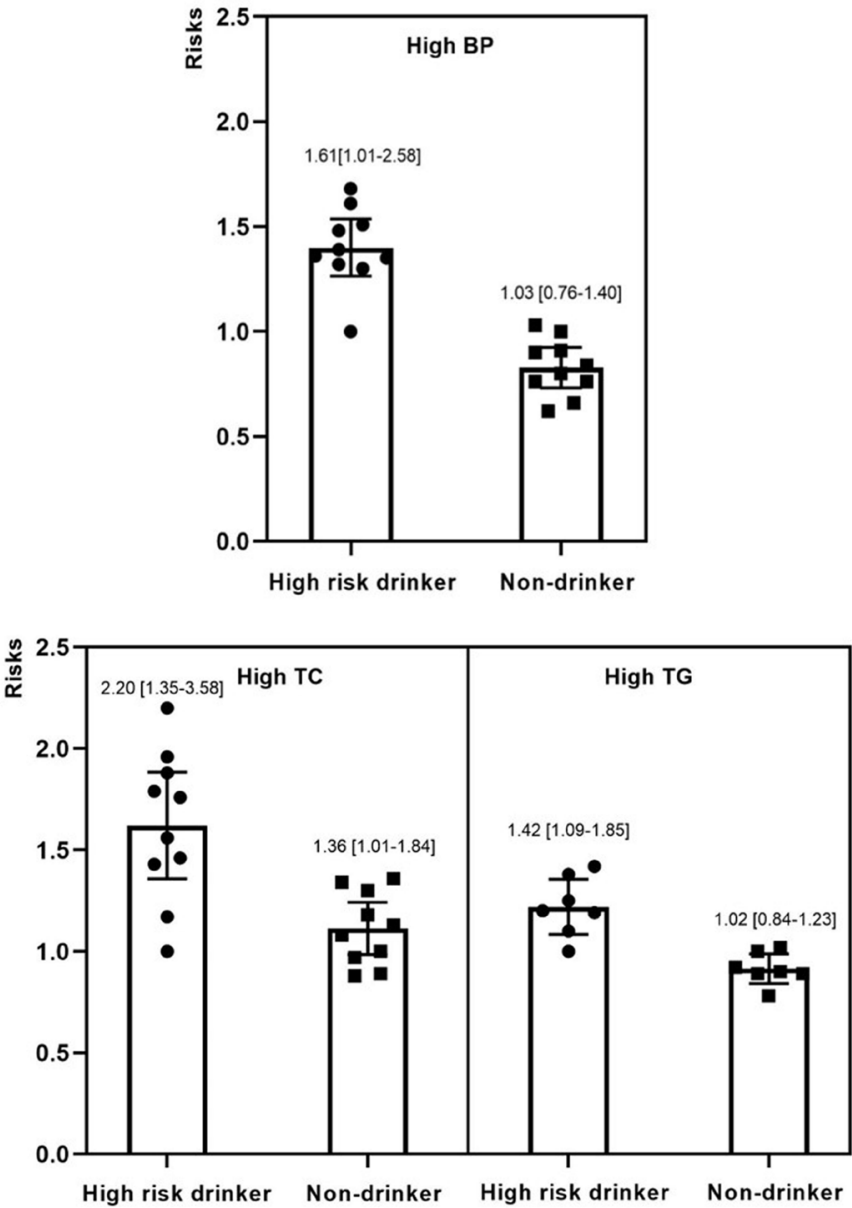

Figure 4 Annual changes in risks of high BP and dyslipidaemia between high risk drinkers ${ }^{\star}$ and non-drinkers $\dagger$ $(n=1330) \neq$. Reference: normal range in each of the variables. ${ }^{*}$ Persistent high-risk drinking for 10 years (twice or more per week, seven or more drinks per drinking on average). $\dagger$ Has been a consistent non-drinker for 10 years. $\ddagger$ Adjusted for age. BP, blood pressure; TC, total cholesterol; TG, triglyceride.

examined the CVD risk factors change by continuous health behaviours among Korean male workers using the NSC 2.0 DB from the NHIS.

Previous studies that attempted various analyses of health behaviours and the prevalence of CVD risk factors among male workers reported the following findings. First, the increase in obesity among Korean adult males is reportedly faster than the average pace of change in Organization for Economic Cooperation and Development (OECD) and other Asian countries. ${ }^{26}$ In particular, the prevalence of obesity among Korean adult males has increased by about 1.3 times in the past 15 years. In 2016, it was estimated that about $41.8 \%$ of adult males were obese. ${ }^{4}$ The results of this study were similar to previous reports from the government. The prevalence of obesity among the cohorts increased by about $8.5 \%$ over the 10 years. Furthermore, the risks of being overweight and of obesity increased by 1.63 and 1.51 times, respectively.
Walking $(\mathrm{N}=1,272)^{*}$

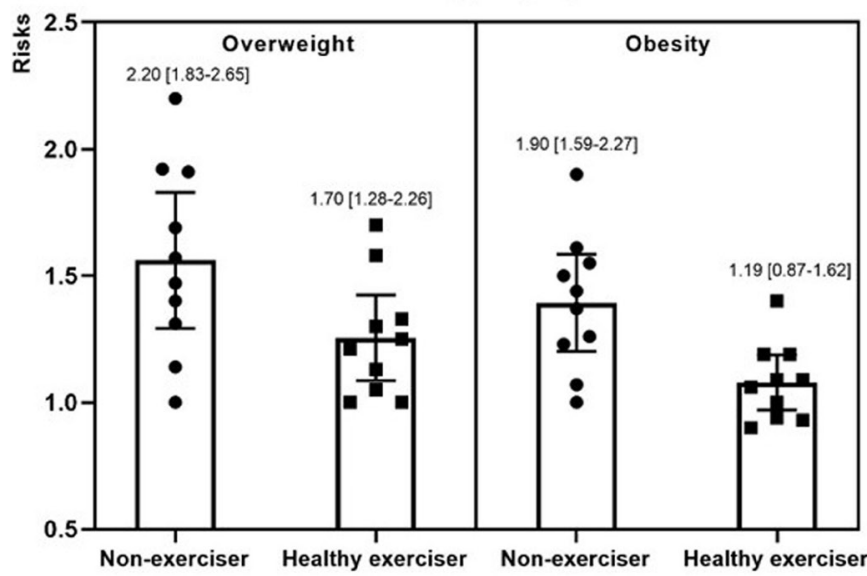

Moderate Activity $(\mathrm{N}=3,670)^{\dagger}$

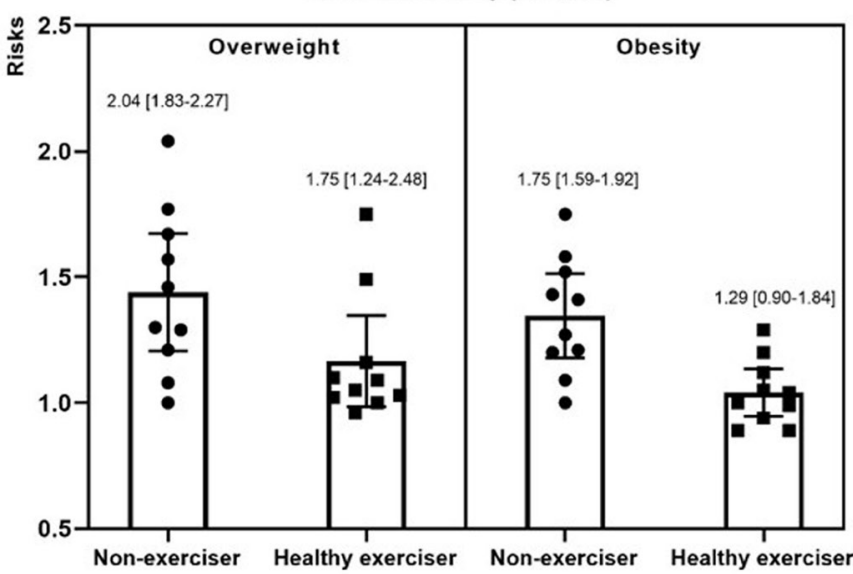

Vigorous Activity $(\mathrm{N}=4,198)^{\ddagger}$

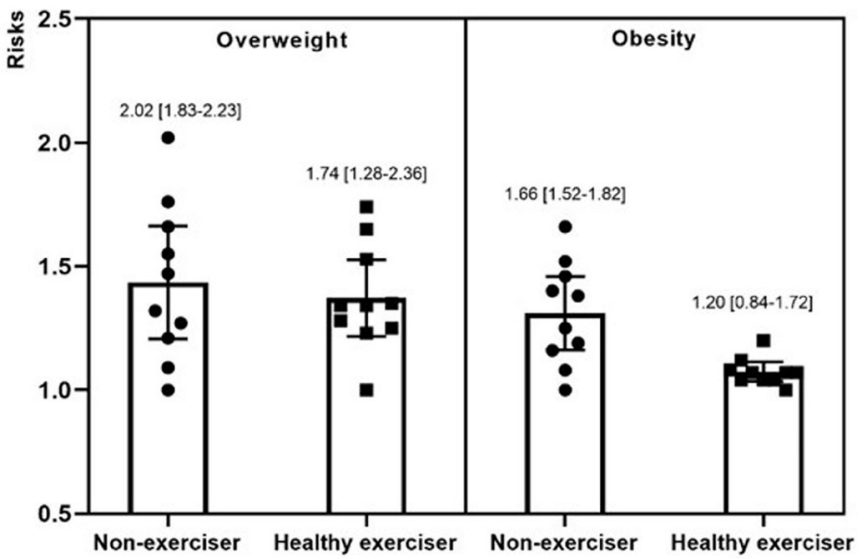

Figure 5 Annual changes in risks of obesity between healthy exercisers and non-exercisers. Reference: normal range in each of the variables. *Adjusted for age, region, income. $†, \ddagger$ Adjusted for age, income.

Second, the prevalence of diabetes in the Korean adult population consistently increased, from $9.1 \%$ in 2005 to $11.3 \%$ in $2016 .{ }^{1}$ The results of the present study showed that the prevalence of pre-diabetes rapidly increased, from $19.64 \%$ to $33.42 \%$, and the risk of high FG increased by about 1.78 times over 10 years. Moreover, the risk of high FG significantly increased in the case of persistent 
smokers over 10 years. Previous studies have reported a dose-response relationship between the amount of smoking and diabetes by insulin resistance.$^{27}$ Other findings have shown that the risk of diabetes among smokers is 1.60 times (95\% CI 1.29 to 1.97 ) higher than nonsmokers in an 8-year longitudinal study. ${ }^{28}$

Third, in 2016, the prevalence of high TC had increased by more than two times since 2005 among the Korean adult population, ${ }^{4}$ and the prevalence of dyslipidaemia in adult males reportedly reached about $48 \%$ in $2016 .{ }^{1}$ This study's results also showed a high prevalence and increasing trend of dyslipidaemia among male workers. Furthermore, the risks of high TC, high TG and high LDL-C increased significantly among persistent smokers. Past findings have reported that the risks of low HDL-C (OR 1.46; 95\% CI 1.19 to 1.80 ), hypertriglyceridaemia (OR 1.41; 95\% CI 1.13 to 1.75 ) and high LDL-C (OR 1.71; $95 \%$ CI 1.18 to 2.48 ) were significantly higher for male smokers than for male non-smokers. ${ }^{29}$ These showed a significant increase of high TC and high TG in high-risk drinkers, consistent with the findings for the Chinese population. ${ }^{30}$

The prevalence of hypertension in domestic workers was $27.3 \%$ in 2016 , which is somewhat lower than the $29.1 \%$ of hypertension prevalence among the entire Korean population. ${ }^{4}$ A past study tracked the international trend of systolic blood pressure (SBP), with the results showing that the average SBP of the male population decreased by about $0.8 \mathrm{~mm} \mathrm{Hg}$, and the ratio of uncontrolled hypertension decreased by about $4 \%$ over 28 years. ${ }^{31}$ Such a trend is more noticeable in higher income countries and is attributable to changes towards healthy behaviours among the overall population as well as to therapeutic interventions. ${ }^{32}$ Nevertheless, the present study found that the prevalence of elevated BP was about $8.68 \%-10.51 \%$ among the participants, except for those previously diagnosed with hypertension or those who were on medication. This indicates that it is necessary to manage the hypertension-risk groups by population based approach for these workers.

The present study is significant for its use of national data and cohort analysis to identify 10-year trends in the medical examination and questionnaire data of domestic male workers. To our knowledge, there have been previous reports for cardiovascular epidemiology of populations of workers in Europe ${ }^{33-35}$ and the USA. ${ }^{36}$ Nevertheless, this study differs in that it followed up the risk factors over 10 years for the same population and analysed the effects of continued health behaviours on the CVD risk factors of workers. Focusing on the CVD risk factors in the working population should not be overlooked with regard to prevention of CVDs. In particular, the study of CVD risk factors is still meaningful in respect of the occurrence of CVDs in the healthy worker group analysed in this study.

However, there are fundamental limitations to this study that precluded classification of several variables and problems of detailed analysis due to restrictions to the secondary analysis of the existing database. For this reason, we could not include more possible risk factors to be considered in our analysis. Furthermore, the consistency in the responses may have suffered to a certain degree because data collection on healthy behaviours included in the Health Questionnaire database relied on individuals' subjective responses and some changes in the physical examination and questionnaire items. Moreover, due to the retrospective nature of the study design, there is an obvious limitation in terms of limited explanatory variables and loss of prospective follow-up. Nonetheless, the present study is a contribution towards deriving national-level findings that can be used as a reference point to propose health policies in the area of health level and risk of CVDs related to the continuous health behaviours of male workers in Korea. The present study is therefore significant because it gives us scientific implication by strongly supporting the findings of previous studies.

\section{CONCLUSIONS}

The present study performed a 10-year retrospective cohort analysis of the cardiovascular risk factors and performance of continuous healthy behaviours among Korean male workers using the NSC 2.0 DB from the NHIS. The analysis showed significant increases in the risk of being obese among male workers over the 10-year period and an overall increase in CVD risk indices with continuous unhealthy behaviours. The results of the study can help in the identification of the risk of CVDs related to health behaviours and can contribute to the proposal of policies for the promotion of workers' health by creating national-level data on the health-level changes of male workers.

Contributors This study was conceived of and planned by all of the authors. HR gave theoretical advice and contributed to the study design, results and interpretation. JJ led in the writing of the manuscript, with contributions and editing from all authors and approval of the final version. JM performed the data collection.

Funding This research was supported by Basic Science Research Program through the National Research Foundation of Korea (NRF) funded by the Ministry of Education (NRF-2019R111A1A01060534) and a Korea University Grant.

Competing interests None declared.

Patient consent for publication Not required.

Ethics approval This study had been approved by the Institutional Review Board (KU-IRB-18-EX-32-A-1) and National Health Insurance Service (NHIS-2018-2-223).

Provenance and peer review Not commissioned; externally peer reviewed.

Data availability statement Data are available on reasonable request. All data are open for accessing and analysing following the approval of National Health Insurance Data Sharing Service.

This content has been supplied by the author(s). It has not been vetted by BMJ Publishing Group Limited (BMJ) and may not have been peer-reviewed. Any opinions or recommendations discussed are solely those of the author(s) and are not endorsed by BMJ. BMJ disclaims all liability and responsibility arising from any reliance placed on the content. Where the content includes any translated material, BMJ does not warrant the accuracy and reliability of the translations (including but not limited to local regulations, clinical guidelines, terminology, drug names and drug dosages), and is not responsible for any error and/or omissions arising from translation and adaptation or otherwise.

Open access This is an open access article distributed in accordance with the Creative Commons Attribution Non Commercial (CC BY-NC 4.0) license, which 
permits others to distribute, remix, adapt, build upon this work non-commercially, and license their derivative works on different terms, provided the original work is properly cited, appropriate credit is given, any changes made indicated, and the use is non-commercial. See: http://creativecommons.org/licenses/by-nc/4.0/.

\section{ORCID iD}

Jiyeon Jung http://orcid.org/0000-0002-8295-3424

\section{REFERENCES}

1 Korea Center for Disease Control and Prevention. The National health statistics 2016. Cheonju, 2016.

2 Alwan A. Global status report on noncommunicable diseases 2010. Geneva: WHO Press, 2011.

3 Tsutsumi A. Prevention and management of work-related cardiovascular disorders. Int J Occup Med Environ Health 2015;28:4-7.

4 Korea center for Disease Control and Prevention. Fact book of chronic diseases 2018. Cheonju, 2018.

$5 \mathrm{Kim} \mathrm{JH}$, Chang SJ. The relation between job stress and health problem according to working condition. Health and Social Science 2012;31:5-24.

6 Ryu H, Jung J, Cho J, et al. Program development and effectiveness of workplace health promotion program for preventing metabolic syndrome among office workers. Int J Environ Res Public Health 2017; $14: 878$

7 Ryu H, Moon J, Jung J. Sex differences in cardiovascular disease risk by socioeconomic status (SES) of workers using National health information database. Int J Environ Res Public Health 2020;17:2047.

8 Asia Pacific Cohort Studies Collaboration. A comparison of the associations between risk factors and cardiovascular disease in Asia and Australasia. Eur J Cardiovasc Prev Rehabil 2005;12:484-91.

9 Larsson SC, Tektonidis TG, Gigante B, et al. Healthy lifestyle and risk of heart failure: results from 2 prospective cohort studies. Circ Heart Fail 2016;9:e002855

10 Eguchi E, Iso H, Tanabe N, et al. Healthy lifestyle behaviours and cardiovascular mortality among Japanese men and women: the Japan collaborative cohort study. Eur Heart $J$ 2012;33:467-77.

11 Rhee CW, Kim JY, Park BJ, et al. Impact of individual and combined health behaviors on all causes of premature mortality among middle aged men in Korea: the Seoul male cohort study. J Prev Med Public Health 2012;45:14-20.

12 Kim JY, Ko Y-J, Rhee CW, et al. Cardiovascular health metrics and all-cause and cardiovascular disease mortality among middle-aged men in Korea: the Seoul male cohort study. J Prev Med Public Health 2013;46:319-28.

13 Jeong H-S. Korea's National Health Insurance-lessons from the past three decades. Health Aff 2011;30:136-44.

14 Ryu D-R. Introduction to the medical research using National health insurance claims database. The Ewha Medical Journal 2017:40:66-77.

15 National Health Insurance Service. National medical examination sample cohort (NHIS-NMES) user manual, 2017. Available: https:// nhiss.nhis.or.kr/bd/ab/bdaba006cv.do [Accessed February 10, 2020]

16 Lee J, Lee JS, Park S-H, et al. Cohort profile: the National health insurance service-national sample cohort (NHIS-NSC), South Korea Int J Epidemiol 2017;46:e15.

17 Lloyd-Jones DM, Hong Y, Labarthe D, et al. Defining and setting national goals for cardiovascular health promotion and disease reduction: the American heart association's strategic impact goal through 2020 and beyond. Circulation 2010;121:586-613.
18 World Health Organization. International association for the study of obesity, International obesity task force. The Asia-Pacific perspective: redefining obesity and its treatment. Sydney: Health Communications, 2000.

19 Yoon YS, Oh SW. Optimal waist circumference cutoff values for the diagnosis of abdominal obesity in Korean adults. Endocrinol Metab 2014;29:418-26.

20 Lee H-Y. New definition for hypertension. J Korean Med Assoc 2018;61:485-92.

21 American Diabetes Association. Diagnosis and classification of diabetes mellitus. Diabetes Care 2007;30 Suppl 1:S42-7.

22 National Cholesterol Education Program (NCEP) Expert Panel on Detection, Evaluation, and Treatment of High Blood Cholesterol in Adults (Adult Treatment Panel III). Third report of the National cholesterol education program (NCEP) expert panel on detection, evaluation, and treatment of high blood cholesterol in adults (adult treatment panel III) final report. Circulation 2002;106:3143-421.

23 Committee for Guidelines for Management of Dyslipidemia. 2015 Korean guidelines for management of dyslipidemia. J Lipid Atheroscler 2015;4:61-92.

24 Korea Center for Disease Control and Prevention, The seventh stage. Korea National health and nutrition examination survey (KNHANES). Cheongju, 2016

25 Oh Y. An econometric analysis of the difference between exercisers and non-exercisers in medical care use. Health and Social Welfare Review 2013;33:471-96.

26 Organization for Economic Cooperation and Development. OECD obesity update 2017. Paris; 2017. https://www.oecd.org/health/ obesity-update.htm [Accessed October 27, 2020].

27 Kim S-K. Cigarette smoking and diabetes. Korean Clin Diabetes 2009;10:73-6.

28 Hur NW, Kim HC, Mo Nam C, et al. Smoking cessation and risk of type 2 diabetes mellitus: Korea medical insurance Corporation study. Eur J Cardiovasc Prev Rehabil 2007;14:244-9.

29 Shin DY, Jang YK, Lee JH, et al. Relationship with smoking and dyslipidemia in Korean adults. J Korean Soc Res Nicotine Tob 2017;8:73-9.

30 Shen Z, Munker S, Wang C, et al. Association between alcohol intake, overweight, and serum lipid levels and the risk analysis associated with the development of dyslipidemia. J Clin Lipidol 2014;8:273-8.

31 Danaei G, Finucane MM, Lin JK, et al. National, regional, and global trends in systolic blood pressure since 1980: systematic analysis of health examination surveys and epidemiological studies with 786 country-years and 5.4 million participants. Lancet 2011;377:568-77.

32 Egan BM, Zhao Y, Axon RN. Us trends in prevalence, awareness, treatment, and control of hypertension, 1988-2008. JAMA 2010;303:2043-50.

33 Puddu PE. Are healthy workers made equal to the general young to middle-aged population in Spain, a southern European country? Eur J Prev Cardiol 2019;71:204748731988747.

34 Fernández-Labandera C, Calvo-Bonacho E, Valdivielso P, et al. Prediction of fatal and non-fatal cardiovascular events in young and middle-aged healthy workers: the IberScore model. Eur J Prev Cardiol 2019;21:204748731989488.

35 Veronesi G, Borchini R, Landsbergis P, et al. Cardiovascular disease prevention at the workplace: assessing the prognostic value of lifestyle risk factors and job-related conditions. Int J Public Health 2018;63:723-32

36 MacDonald LA, Bertke S, Hein MJ, et al. Prevalence of cardiovascular health by occupation: a cross-sectional analysis among U.S. workers aged $\geq 45$ years. Am J Prev Med 2017:53:152-61. 